\title{
Dimorfismo sexual em estudo do crânio, sacro e pelve de brasileiros
}

\author{
Sexual dimorphism in study of brazilian skull, sacro and pelve
}

Graciele Dib Nunes Silva CRisthiane Martins SCHMidT ${ }^{1}$ Viviane Ulbricht ${ }^{\mathrm{I}}$ Francisco Carlos Groppo EdUARDo DARUge JUNIOR ${ }^{\mathrm{I}}$ Luiz Francesquini Junior JoÃo CESAR BARbIERI BEDRAN DE CASTRO ${ }^{1}$

${ }^{\mathrm{I}}$ Faculdade de Odontologia de Piracicaba (UNICAMP). Piracicaba/ SP - Brasil
RESUmo Este estudo analítico observacional tem a finalidade de verificar a presença de dimorfismo sexual em ossadas humanas brasileiras por meio de medidas lineares realizadas no crânio (o losango formado pelo forame incisivo - forame palatino maior (direito e esquerdo) e forame palatino maior - básio (direito e esquerdo), pelve (diâmetro vertical do acetábulo, comprimento e largura do forame obturado, incisura isquiática maior-medida da corda e medida da profundidade, distância ilíaca anterossuperior do tubérculo púbico) e sacro (largura superior do sacro e comprimento e largura da primeira vértebra sacra) de 191 ossadas de brasileiros, existentes no Biobanco osteológico e tomográfico Prof. Dr. Eduardo Daruge, da FOP/UNICAMP. Foi realizada a análise estatística dos dados pelo teste de Kolmogorov-Smirnov e Levene para avaliar, respectivamente, a normalidade e a homogeneidade das variâncias. Também foram submetidas ao teste $t$ de Student não pareado, tendo sido observado dimorfismo sexual nesses ossos. Com as variáveis diâmetro vertical do acetábulo, comprimento do forame obturado, corda da incisura isquiática maior e Básio até o forame palatino maior, obteve-se fórmula em regressão logística que permite índice $85,7 \%$ de identificação do sexo, ressaltando-se a importância do crânio, do sacro e da pelve no processo de identificação post mortem em ossadas humanas brasileiras.

Palavras-chave: Antropologia Forense. Antropometria. Ossos. Dimorfismo SEXUAL.

ABSTRACT The present observational analytical study has the purpose of verifying the
presence of sexual dimorphism in brazilian human bones by means of linear measure-
ments performed on the skull (the diamond formed by the incisive foramen - palatine
foramen major (right and left) and palatine foramen major - basement (right and left)),
pelvis (vertical diameter of the acetabulum, length and width of the foramen foramen,
ischiatic notch length of the cord and depth measurement, anterior superior iliac dis-
tance of the pubic tubercle) and sacrum (superior width of the sacrum and length and
width of the first sacral vertebra) of 191 bones of Brazilians, existing in the osteological
and tomography Biobank Dr. Eduardo Daruge, of FOP / UNICAMP. Statistical analysis
of the data was performed by the Kolmogorov-Smirnov and Levene test to evaluate,
respectively, the normality and homogeneity of variances. The data were also submitted
to unpaired Student's $t$-test, having been sexual dimorphism observed in these bones.
With the variable's vertical diameter of the acetabulum, length of filled foramen, chor-
dae of the major sciatic notch and Basion to the major palatine foramen, was obtained
a logistic regression formula that allows $85.7 \%$ of gender identification, highlighting the
importance of the skull, sacrum and pelvis in the process of post-mortem identification
in brazilian human bones. Keywords: Forensic Anthropology. Anthropometry. Bones. Sex CharacteRISTICS. 


\section{INTRODUÇÃo}

A antropometria óssea consiste em área da Antropologia Forense, que tem a finalidade (por meio de metodologia quantitativa) de auxiliar no processo de identificação post mortem de um corpo humano (íntegro ou não), sendo considerada como um método secundário, ou seja, embora auxilie no processo de identificação, não permite estabelecer a identidade, que só é admitida pelos métodos primários (exame dactiloscópico, dentário, numeração de próteses metálicas e análise do DNA).

Além das variações tanatológicas referentes aos fenômenos transformativos destrutivos ocorridos após a morte, deve-se ter em consideração outros fatores no processo de identificação, como ataque de animais e produtos químicos, entre outros, os quais irão influenciar a avaliação do método secundário antropométrico. ${ }^{1}$

$\mathrm{Na}$ determinação do sexo, as variações das características da diferenciação sexual óssea sofrem mudanças ao longo dos anos, especialmente por fatores genéticos e hormonais, ${ }^{2}$ seguidos dos fatores ambientais, da dieta, do clima, de doenças crônicas e do trabalho, entre outros. ${ }^{3}$

Sabe-se que os esqueletos de crianças ( 0 a 12 anos de idade) possuem características qualitativas e quantitativas pouco pronunciadas, dificultando a determinação do sexo. Após a puberdade e sob a influência dos hormônios inerentes a cada sexo e do meio ambiente, o esqueleto desenvolve características diferenciais, como, proeminências, rugosidades, cristas, apófises, saliências mais destacadas para o homem e mais discretas e sutis para a mulher. ${ }^{4}$

Segundo Coma (1999), ${ }^{5}$ de $10 \%$ a $20 \%$ dos europeus apresentam características sexuais dimórficas mescladas, que trazem confusão quando dos exames. É o caso do pseudo-hermafrodita, o hermafrodita puro, o transexual que utiliza os recursos cirúrgicos para modificar características ósseas, hormonais e sexuais e dos indivíduos que, embora mantenham o sexo compatível com o seu gênero, têm tamanho ósseo compatível ao sexo oposto.

Já está consagrado na literatura que os ossos da pelve, crânio e mandíbula são os que apresentam características qualitativas e quantitativas confiáveis para a determinação do sexo e, com base nesses estudos, se desenvolveram modelos matemáticos específicos para cada osso ou grupo de ossos, visando determinar o sexo com a menor porcentagem de erro. ${ }^{6}$

O presente estudo visou convalidar a importância dos ossos da pelve, crânio e mandíbula na identificação da população brasileira, de predominância étnica faioderma e leucoderma, visando determinar o dimorfismo das medidas avaliadas, bem como, criar um modelo matemático para determinar o sexo com exatidão.

\section{MAterial e Métodos}

\section{Amostra}

Esta pesquisa foi submetida e aprovada pelo Comitê de Ética em Pesquisa da Faculdade de Odontologia de Piracicaba - Universidade de Campinas, sob o protocolo $\mathrm{n}^{\circ}$. 138/2014(CAAE 38522714.6.0000.5418). Foi realizada em 191 ossadas, sendo 109 masculinas e 82 femininas, pertencentes ao Biobanco osteológico e tomográfico Prof. Dr. Eduardo Daruge, da FOP/ UNICAMP, com procedência conhecida e de absoluta certeza quanto à idade, sexo e ancestralidade. As medições foram feitas somente em ossos íntegros.

Para a realização das medidas, utilizou-se paquímetro digital de precisão marca Stainless - hardened ${ }^{\circledR}$ 150 mm Mauá (São Paulo Brasil), observando-se que a cada medida as pontas do aparelho eram unidas e o calibrador deixado no número zero.

As medidas realizadas foram:

- na pelve: diâmetro vertical do acetábulo, comprimento e largura do forame obturado, incisura isquiática maior-medida da corda e medida da profundidade, distância ilíaca anterossuperior do tubérculo púbico;

- no sacro: largura superior do sacro e comprimento e largura da primeira vértebra sacral;

- no crânio: o losango formado pelo forame incisivo - forame palatino maior (direito e esquerdo) e forame palatino maior - básio (direito e esquerdo).

\section{Calibração}

Para iniciar as medições, promoveu-se previamente a calibração inter e intra-examinador, sendo realizada em três períodos distintos em 25 ossadas, com intervalo entre elas de um mês, de forma a se aplicar a análise de correlação intraclasse para as avaliações inter e intra-examinador.

Foi utilizado o coeficiente de correlação intraclasse, segundo Szklo e Nieto (2000), para interpretação do coeficiente obtido, que foi o ICC 0,9593 e p< 0,0001 , considerado excelente.

\section{Análise Estatística}

Foi realizado o teste de Kolmorow-Smirnov e Levene para avaliar a homogeneidade e normalidade, também se utilizou o teste $t$ de Student, teste de Pear- 
son e método Stepwise-Wald. Na análise estatística, foi usado o software GRAPHPAD PRISM 7.0.

As medidas obtidas foram submetidas ao teste $t$ de Student não pareado para a análise da diferença estatisticamente relevante entre as médias das medidas realizadas nas ossadas do sexo masculino e feminino (Tabela 1).

Foram também submetidas ao teste de Pearson para a análise da correlação entre as variáveis (Tabela 2), bem como, a correlação entre o sexo feminino (Tabela 3) e o sexo masculino (Tabela 4).

Posteriormente, pelo método Stepwise - Wald obtém-se as medidas a serem utilizadas para a fórmula de regressão logística (Tabela 5).
Aplica-se o modelo de regressão logística à amostra e considerando o sexo masculino como " 1 " e o feminino como "0" para efeito do cálculo, obtém-se o percentual de predição do sexo (Tabela 6).

Para a análise dos resultados, está definido para este estudo o nível de significância de 0,05 (5\%), ou seja, quando $\mathrm{p}<0,05$, a diferença é estatisticamente relevante; se $\mathrm{p}>0,05$, a diferença é estatisticamente irrelevante.

\section{Resultados}

Realizada as medições, os resultados foram compilados nas tabelas a seguir.

Tabela 1. Teste $t$-Student com média das medidas masculinas e femininas em milímetros e desvio padrão.

\begin{tabular}{|c|c|c|c|}
\hline Variáveis & Sexo & $\begin{array}{l}\text { Média } \\
\text { ( } \pm \text { erro padrão) }\end{array}$ & $\mathrm{p}$ \\
\hline \multirow{2}{*}{ Diâmetro vertical do acetábulo } & Masculino $(n=108)$ & $51.6( \pm 0.3)$ & \multirow{2}{*}{$<0.0001$} \\
\hline & Feminino $(\mathrm{n}=79)$ & $46.6( \pm 0.35)$ & \\
\hline \multirow{2}{*}{ Comprimento do forame obturado } & Masculino (n=109) & $51.1( \pm 0.38)$ & \multirow{2}{*}{$<0.0001$} \\
\hline & Feminino $(\mathrm{n}=82)$ & $46.7( \pm 0.46)$ & \\
\hline \multirow{2}{*}{ Largura do Forame obturado } & Masculino (n=109) & $32.3( \pm 0.29)$ & \multirow{2}{*}{0.29} \\
\hline & Feminino $(\mathrm{n}=80)$ & $31.8( \pm 0.36)$ & \\
\hline \multirow{2}{*}{ Distância ilíaca anterossuperior do tubérculo púbico } & Masculino $(\mathrm{n}=109)$ & $125.7( \pm 0.83)$ & \multirow{2}{*}{0.87} \\
\hline & Feminino $(\mathrm{n}=82)$ & $125.9( \pm 1.16)$ & \\
\hline \multirow{2}{*}{ Incisura isquiática maior - Corda } & Masculino $(n=106)$ & $106.4( \pm 0.86)$ & \multirow{2}{*}{0.87} \\
\hline & Feminino $(\mathrm{n}=78)$ & $106.2( \pm 0.88)$ & \\
\hline \multirow{2}{*}{ Incisura isquiática Maior - Profundidade } & Masculino (n=107) & $49.9( \pm 0.54)$ & \multirow{2}{*}{0.0031} \\
\hline & Feminino $(\mathrm{n}=80)$ & $47.6( \pm 0.53)$ & \\
\hline \multirow{2}{*}{ Largura superior sacro } & Masculino $(n=106)$ & $30.7( \pm 0.25)$ & \multirow{2}{*}{$<0.0001$} \\
\hline & Feminino $(\mathrm{n}=79)$ & $28.1( \pm 0.33)$ & \\
\hline \multirow[t]{2}{*}{ Comprimento da primeira vértebra sacra } & Masculino (n=99) & $39( \pm 0.28)$ & \multirow{2}{*}{0.0124} \\
\hline & Feminino $(\mathrm{n}=78)$ & $38( \pm 0.3)$ & \\
\hline \multirow{2}{*}{ Largura da primeira vértebra sacra } & Masculino (n=99) & $39.2( \pm 0.28)$ & \multirow{2}{*}{0.0026} \\
\hline & Feminino $(\mathrm{n}=78)$ & $37.9( \pm 0.31)$ & \\
\hline \multirow{2}{*}{ Forame incisivo até o forame palatino maior - esquerdo } & Masculino (n=97) & $50( \pm 0.37)$ & \multirow{2}{*}{$<0.0001$} \\
\hline & Feminino $(\mathrm{n}=78)$ & $47.6( \pm 0.4)$ & \\
\hline \multirow{2}{*}{ Forame incisivo até o forame palatino maior - direito } & Masculino (n=97) & $50.2( \pm 0.4)$ & \multirow{2}{*}{$<0.0001$} \\
\hline & Feminino $(\mathrm{n}=78)$ & $47.8( \pm 0.4)$ & \\
\hline \multirow{2}{*}{ Forame incisivo até o forame palatino maior - média } & Masculino (n=97) & $50.1( \pm 0.38)$ & \multirow[t]{2}{*}{$<0.0001$} \\
\hline & Feminino $(\mathrm{n}=78)$ & $47.7( \pm 0.39)$ & \\
\hline \multirow[t]{2}{*}{ Básio até o forame palatino maior - esquerdo } & Masculino (n=109) & $43.9( \pm 0.54)$ & \multirow{2}{*}{$<0.0001$} \\
\hline & Feminino $(\mathrm{n}=82)$ & $48.5( \pm 0.84)$ & \\
\hline \multirow[t]{2}{*}{ Básio até o forame palatino maior - direito } & Masculino (n=109) & $35.2( \pm 0.42)$ & \multirow[t]{2}{*}{0.0165} \\
\hline & Feminino $(\mathrm{n}=82)$ & $33.6( \pm 0.49)$ & \\
\hline \multirow{2}{*}{ Básio até o forame palatino maior - média } & Masculino (n=109) & $39.5( \pm 0.37)$ & \multirow{2}{*}{0.012} \\
\hline & Feminino $(n=82)$ & $41.1( \pm 0.52)$ & \\
\hline
\end{tabular}


A Tabela 1 revela que todas as variáveis, com exceção da largura do forame obturado, distância ilíaca anterossuperior do tubérculo púbico e da corda da incisura isquiática maior, mostraram diferenças estatisticamente significantes entre os sexos $(\mathrm{p}<0,05)$.

Demonstra também que com exceção das medidas referentes à distância do básio até o forame palatino maior do lado esquerdo e a média entre os lados esquerdo e direito dessa medida, em todas as demais, a média do sexo masculino é maior que a do sexo feminino.

A Tabela 2 demonstra que, embora muitas correlações tenham se mostrado significativas $(\mathrm{p}<0.05)$, poucas podem ser consideradas importantes. Além das correlações óbvias entre as medidas médias e os valores que as geraram ou as medidas tomadas no mesmo osso, houve correlação moderada entre o diâmetro vertical do acetábulo com o comprimento do forame obturado, com a profundidade da incisura isquiática maior e com a largura superior sacro, sendo que as últimas também apresentaram correlação moderada.

Tabela 2. Coeficiente de correlação de Pearson da média das medidas do diâmetro vertical do acetábulo com as médias das demais medidas estudadas.

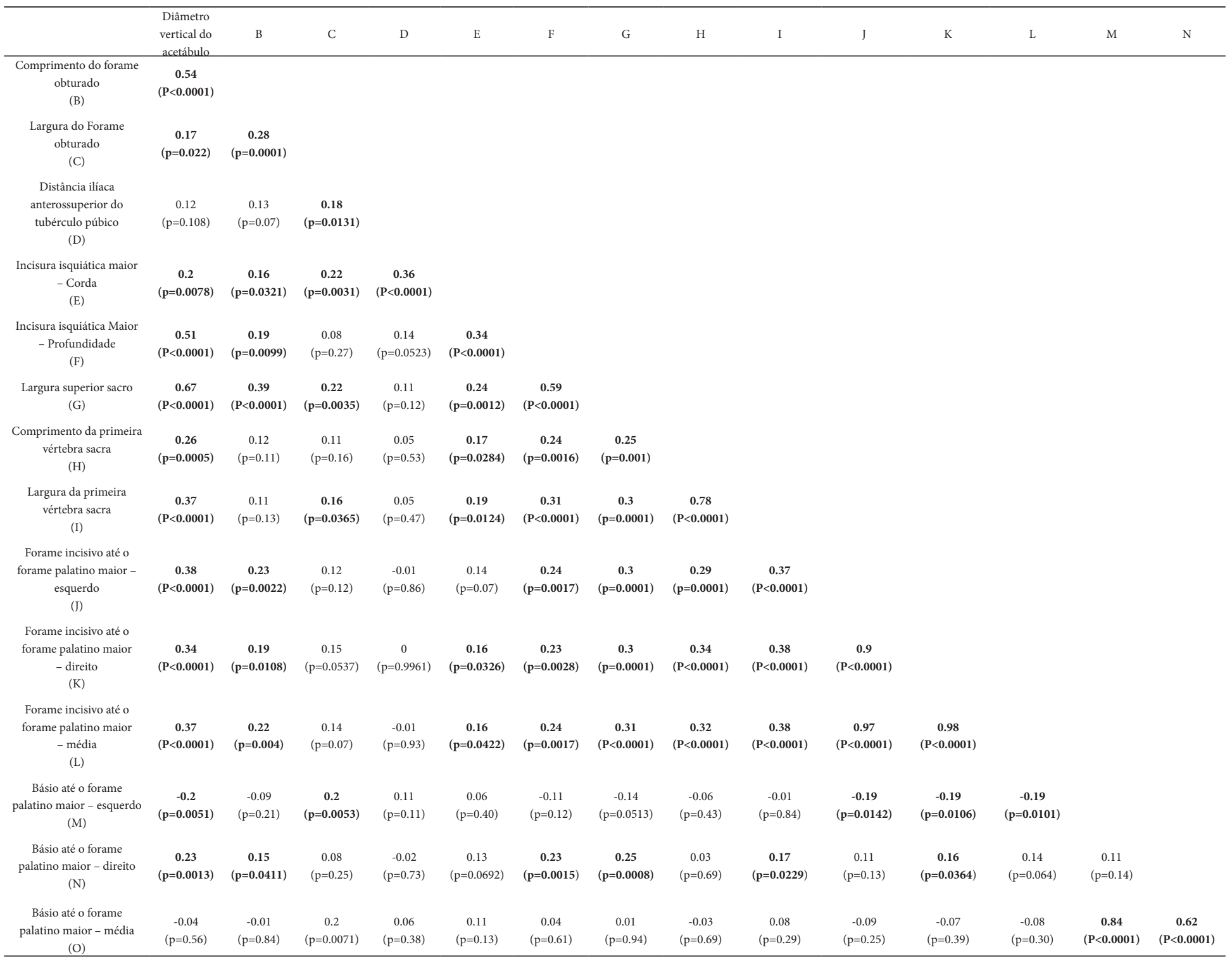


Tabela 3. Coeficiente de correlação de Pearson da média das medidas do diâmetro vertical do acetábulo com as médias das demais medidas estudadas para o sexo feminino.

\begin{tabular}{|c|c|c|c|c|c|c|c|c|c|c|c|c|c|c|}
\hline Feminino & $\begin{array}{c}\text { Diàmetro } \\
\text { vertical do } \\
\text { acetábulo }\end{array}$ & в & c & D & $\mathrm{E}$ & $\mathrm{F}$ & G & H & I & J & к & L & M & $\mathrm{N}$ \\
\hline $\begin{array}{l}\text { Comprimento do forame obturado } \\
\text { (B) }\end{array}$ & $\begin{array}{c}0.44 \\
(\mathrm{p}=0.0001)\end{array}$ & & & & & & & & & & & & & \\
\hline $\begin{array}{l}\text { Largura do Forame obturado } \\
\text { (C) }\end{array}$ & $\begin{array}{c}0.34 \\
(\mathrm{p}=0.0028)\end{array}$ & $\begin{array}{c}0.2 \\
(\mathrm{p}=0.0712)\end{array}$ & & & & & & & & & & & & \\
\hline $\begin{array}{l}\text { Distância ilíaca anterossuperior do tubérculo púbico } \\
\text { (D) }\end{array}$ & $\begin{array}{c}0.14 \\
(\mathrm{p}=0.2058)\end{array}$ & $\begin{array}{c}0.15 \\
(\mathrm{p}=0.1814)\end{array}$ & $\begin{array}{c}0.29 \\
(\mathrm{p}=0.0081)\end{array}$ & & & & & & & & & & & \\
\hline $\begin{array}{l}\text { Incisura isquiática maior - Corda } \\
\text { (E) }\end{array}$ & $\begin{array}{c}0.34 \\
(\mathrm{p}=0.0027)\end{array}$ & $\begin{array}{c}0.11 \\
(\mathrm{p}=0.3568)\end{array}$ & $\begin{array}{c}0.29 \\
(\mathrm{p}=0.0105)\end{array}$ & $\begin{array}{c}0.24 \\
(\mathrm{p}=0.032)\end{array}$ & & & & & & & & & & \\
\hline $\begin{array}{l}\text { Incisura isquiática Maior - Profundidade } \\
\text { (F) }\end{array}$ & $\begin{array}{c}0.31 \\
(\mathrm{p}=0.0058)\end{array}$ & $\begin{array}{c}-0.2 \\
(\mathrm{p}=0.0761)\end{array}$ & $\begin{array}{c}0.04 \\
(\mathrm{p}=0.7237)\end{array}$ & $\begin{array}{c}0.01 \\
(\mathrm{p}=0.9538)\end{array}$ & $\begin{array}{c}0.17 \\
(\mathrm{p}=0.1315)\end{array}$ & & & & & & & & & \\
\hline $\begin{array}{l}\text { Largura superior sacro } \\
\qquad \text { (G) }\end{array}$ & $\begin{array}{c}0.59 \\
(\mathrm{P}<0.0001)\end{array}$ & $\begin{array}{c}0.14 \\
(\mathrm{p}=0.2045)\end{array}$ & $\begin{array}{c}0.18 \\
(\mathrm{p}=0.1112)\end{array}$ & $\begin{array}{c}0.05 \\
(\mathrm{p}=0.6781)\end{array}$ & $\begin{array}{c}0.33 \\
(\mathrm{p}=0.0032)\end{array}$ & $\begin{array}{c}0.54 \\
(\mathrm{P}<0.0001)\end{array}$ & & & & & & & & \\
\hline $\begin{array}{l}\text { Comprimento da primeira vértebra sacra } \\
\qquad \begin{array}{l}\text { (H) }\end{array}\end{array}$ & $\begin{array}{c}0.12 \\
(\mathrm{p}=0.3133)\end{array}$ & $\begin{array}{c}-0.07 \\
(\mathrm{p}=0.5606)\end{array}$ & $\begin{array}{c}0.06 \\
(\mathrm{p}=0.605)\end{array}$ & $\begin{array}{c}0.09 \\
(\mathrm{p}=0.414)\end{array}$ & $\begin{array}{c}0.28 \\
(\mathrm{p}=0.0169)\end{array}$ & $\begin{array}{c}0.16 \\
(\mathrm{p}=0.1641)\end{array}$ & $\begin{array}{c}0.22 \\
(\mathrm{p}=0.0625)\end{array}$ & & & & & & & \\
\hline $\begin{array}{l}\text { Largura da primeira vértebra sacra } \\
\text { (I) }\end{array}$ & $\begin{array}{c}0.25 \\
(\mathrm{p}=0.0333)\end{array}$ & $\begin{array}{c}-0.08 \\
(\mathrm{p}=0.4688)\end{array}$ & $\begin{array}{c}0.15 \\
(\mathrm{p}=0.1965)\end{array}$ & $\begin{array}{c}0.08 \\
(\mathrm{p}=0.4642)\end{array}$ & $\begin{array}{c}0.22 \\
(\mathrm{p}=0.0589)\end{array}$ & $\begin{array}{c}0.24 \\
(\mathrm{p}=0.0373)\end{array}$ & $\begin{array}{c}0.34 \\
(\mathrm{p}=0.0031)\end{array}$ & $\begin{array}{c}0.8 \\
(\mathrm{P}<0.0001)\end{array}$ & & & & & & \\
\hline $\begin{array}{l}\text { Forame incisivo até o forame palatino maior } \\
\text { - esquerdo } \\
\text { (J) }\end{array}$ & $\begin{array}{c}0.4 \\
(\mathrm{p}=0.0003)\end{array}$ & $\begin{array}{c}0.28 \\
(\mathrm{p}=0.0143)\end{array}$ & $\begin{array}{c}0.05 \\
(\mathrm{p}=0.6484)\end{array}$ & $\begin{array}{c}-0.06 \\
(\mathrm{p}=0.5769)\end{array}$ & $\begin{array}{c}0.22 \\
(\mathrm{p}=0.0621)\end{array}$ & $\begin{array}{c}0.29 \\
(\mathrm{p}=0.0122)\end{array}$ & $\begin{array}{c}0.33 \\
(\mathrm{p}=0.0043)\end{array}$ & $\begin{array}{c}0.27 \\
(\mathrm{p}=0.0179)\end{array}$ & $\begin{array}{c}0.37 \\
(\mathrm{p}=0.0009)\end{array}$ & & & & & \\
\hline $\begin{array}{l}\text { Forame incisivo até o forame palatino maior } \\
\text { - direito } \\
\text { (K) }\end{array}$ & $\begin{array}{c}0.35 \\
(\mathrm{p}=0.0021)\end{array}$ & $\begin{array}{c}0.24 \\
(\mathrm{p}=0.0311)\end{array}$ & $\begin{array}{c}-0.04 \\
(\mathrm{p}=0.7498)\end{array}$ & $\begin{array}{c}-0.06 \\
(\mathrm{p}=0.595)\end{array}$ & $\begin{array}{c}0.2 \\
(\mathrm{p}=0.0957)\end{array}$ & $\begin{array}{c}0.3 \\
(\mathrm{p}=0.009)\end{array}$ & $\begin{array}{c}0.36 \\
(\mathrm{p}=0.0015)\end{array}$ & $\begin{array}{c}0.32 \\
(\mathrm{p}=0.0037)\end{array}$ & $\begin{array}{c}0.35 \\
(\mathrm{p}=0.0015)\end{array}$ & $\begin{array}{c}0.91 \\
(\mathrm{P}<0.0001)\end{array}$ & & & & \\
\hline $\begin{array}{l}\text { Forame incisivo até o forame palatino maior } \\
\text { - média } \\
\text { (L) }\end{array}$ & $\begin{array}{c}0.39 \\
(\mathrm{p}=0.0006)\end{array}$ & $\begin{array}{c}0.27 \\
(\mathrm{p}=0.0185)\end{array}$ & $\begin{array}{c}0.01 \\
(\mathrm{p}=0.944)\end{array}$ & $\begin{array}{c}-0.06 \\
(\mathrm{p}=0.5777)\end{array}$ & $\begin{array}{c}0.21 \\
(\mathrm{p}=0.0713)\end{array}$ & $\begin{array}{c}0.3 \\
(\mathrm{p}=0.0089)\end{array}$ & $\begin{array}{c}0.35 \\
(\mathrm{p}=0.002)\end{array}$ & $\begin{array}{c}0.3 \\
(\mathrm{p}=0.0071)\end{array}$ & $\begin{array}{c}0.37 \\
(\mathrm{p}=0.0009)\end{array}$ & $\begin{array}{c}0.98 \\
(\mathrm{P}<0.0001)\end{array}$ & $\begin{array}{c}0.98 \\
(\mathrm{P}<0.0001)\end{array}$ & & & \\
\hline $\begin{array}{l}\text { Básio até o forame palatino maior - esquerdo } \\
\qquad(\mathrm{M})\end{array}$ & $\begin{array}{c}0.11 \\
(\mathrm{p}=0.3311)\end{array}$ & $\begin{array}{c}0.07 \\
(\mathrm{p}=0.5156)\end{array}$ & $\begin{array}{c}0.25 \\
(\mathrm{p}=0.024)\end{array}$ & $\begin{array}{c}0.3 \\
(\mathrm{p}=0.0064)\end{array}$ & $\begin{array}{c}0.15 \\
(\mathrm{p}=0.2014)\end{array}$ & $\begin{array}{c}0.06 \\
(\mathrm{p}=0.6038)\end{array}$ & $\begin{array}{c}0.12 \\
(\mathrm{p}=0.286)\end{array}$ & $\begin{array}{c}0 \\
(\mathrm{p}=0.9709)\end{array}$ & $\begin{array}{c}0.1 \\
(\mathrm{p}=0.3828)\end{array}$ & $\begin{array}{c}0.01 \\
(\mathrm{p}=0.9497)\end{array}$ & $\begin{array}{c}-0.07 \\
(\mathrm{p}=0.5334)\end{array}$ & $\begin{array}{c}-0.03 \\
(\mathrm{p}=0.7753)\end{array}$ & & \\
\hline $\begin{array}{l}\text { Básio atéo oforame palatino maior - direito } \\
\qquad(\mathrm{N})\end{array}$ & $\begin{array}{c}0.06 \\
(\mathrm{p}=0.6116)\end{array}$ & $\begin{array}{c}-0.13 \\
(\mathrm{p}=0.2268)\end{array}$ & $\begin{array}{c}-0.01 \\
(\mathrm{p}=0.948)\end{array}$ & $\begin{array}{c}-0.1 \\
(\mathrm{p}=0.354)\end{array}$ & $\begin{array}{c}0.03 \\
(\mathrm{p}=0.7803)\end{array}$ & $\begin{array}{c}0.08 \\
(\mathrm{p}=0.4645)\end{array}$ & $\begin{array}{c}0.17 \\
(\mathrm{p}=0.1293)\end{array}$ & $\begin{array}{c}0.07 \\
(\mathrm{p}=0.564)\end{array}$ & $\begin{array}{c}0.2 \\
(\mathrm{p}=0.083)\end{array}$ & $\begin{array}{c}0.06 \\
(\mathrm{p}=0.6277)\end{array}$ & $\begin{array}{c}0.08 \\
(\mathrm{p}=0.4937)\end{array}$ & $\begin{array}{c}0.07 \\
(\mathrm{p}=0.5511)\end{array}$ & $\begin{array}{c}0.18 \\
(\mathrm{p}=0.1002)\end{array}$ & \\
\hline $\begin{array}{l}\text { Básio até o forame palatino maior - média } \\
\text { (O) }\end{array}$ & $\begin{array}{c}0.11 \\
(\mathrm{p}=0.3285)\end{array}$ & $\begin{array}{c}-0.04 \\
(\mathrm{p}=0.7297)\end{array}$ & $\begin{array}{c}0.18 \\
(\mathrm{p}=0.1089)\end{array}$ & $\begin{array}{c}0.16 \\
(\mathrm{p}=0.1443)\end{array}$ & $\begin{array}{c}0.12 \\
(\mathrm{p}=0.3098)\end{array}$ & $\begin{array}{c}0.12 \\
(\mathrm{p}=0.2958)\end{array}$ & $\begin{array}{c}0.19 \\
(\mathrm{p}=0.0981)\end{array}$ & $\begin{array}{c}0.04 \\
(\mathrm{p}=0.7191)\end{array}$ & $\begin{array}{c}0.18 \\
(\mathrm{p}=0.116)\end{array}$ & $\begin{array}{c}0.04 \\
(\mathrm{p}=0.7142)\end{array}$ & $\begin{array}{c}0 \\
(\mathrm{p}=0.9927)\end{array}$ & $\begin{array}{c}0.02 \\
(\mathrm{p}=0.8555)\end{array}$ & $\begin{array}{c}0.86 \\
(\mathrm{P}<0.0001)\end{array}$ & $\begin{array}{c}0.65 \\
(\mathrm{P}<0.0001)\end{array}$ \\
\hline
\end{tabular}

Tabela 4. Coeficiente de correlação de Pearson da média da medida do diâmetro vertical do acetábulo com as médias das demais medidas estudadas para o sexo masculino.

\begin{tabular}{|c|c|c|c|c|c|c|c|c|c|c|c|c|c|c|}
\hline Masculino & $\begin{array}{c}\text { Diâmetro } \\
\text { vertical do acetábulo }\end{array}$ & B & C & D & $\mathrm{E}$ & $\mathrm{F}$ & G & $\mathrm{H}$ & I & $J$ & $\mathrm{~K}$ & $\mathrm{~L}$ & M & $\mathrm{N}$ \\
\hline $\begin{array}{l}\text { Comprimento do forame obturado } \\
\text { (B) }\end{array}$ & $\begin{array}{c}0.29 \\
(\mathrm{p}=0.002)\end{array}$ & & & & & & & & & & & & & \\
\hline $\begin{array}{l}\text { Largura do Forame obturado } \\
\text { (C) }\end{array}$ & $\begin{array}{c}0.04 \\
(\mathrm{p}=0.7187)\end{array}$ & $\begin{array}{c}0.33 \\
(\mathrm{p}=0.0004)\end{array}$ & & & & & & & & & & & & \\
\hline $\begin{array}{l}\text { Distância iliaca anterossuperior do tubérculo púbico } \\
\text { (D) }\end{array}$ & $\begin{array}{c}0.19 \\
(\mathrm{p}=0.0455)\end{array}$ & $\begin{array}{c}0.16 \\
(\mathrm{p}=0.0865)\end{array}$ & $\begin{array}{c}0.08 \\
(\mathrm{p}=0.4276)\end{array}$ & & & & & & & & & & & \\
\hline $\begin{array}{l}\text { Incisura isquiática maior - Corda } \\
\text { (E) }\end{array}$ & $\begin{array}{c}0.22 \\
(\mathrm{p}=0.0275)\end{array}$ & $\begin{array}{c}0.22 \\
(\mathrm{p}=0.0239)\end{array}$ & $\begin{array}{c}0.17 \\
(\mathrm{p}=0.0847)\end{array}$ & $\begin{array}{c}0.46 \\
(\mathrm{P}<0.0001)\end{array}$ & & & & & & & & & & \\
\hline $\begin{array}{l}\text { Incisura isquiática Maior - Profundidade } \\
\qquad \text { (F) }\end{array}$ & $\begin{array}{c}0.57 \\
(\mathrm{P}<0.0001)\end{array}$ & $\begin{array}{c}0.29 \\
(\mathrm{p}=0.002)\end{array}$ & $\begin{array}{c}0.08 \\
(\mathrm{p}=0.425)\end{array}$ & $\begin{array}{c}0.25 \\
(\mathrm{p}=0.0087)\end{array}$ & $\begin{array}{c}0.44 \\
(\mathrm{P}<0.0001)\end{array}$ & & & & & & & & & \\
\hline $\begin{array}{l}\text { Largura superior sacro } \\
\text { (G) }\end{array}$ & $\begin{array}{c}0.53 \\
(\mathrm{P}<0.0001)\end{array}$ & $\begin{array}{c}0.31 \\
(\mathrm{p}=0.0013)\end{array}$ & $\begin{array}{c}0.21 \\
(\mathrm{p}=0.0324)\end{array}$ & $\begin{array}{c}0.2 \\
(\mathrm{p}=0.0447)\end{array}$ & $\begin{array}{c}0.2 \\
(\mathrm{p}=0.0363)\end{array}$ & $\begin{array}{c}0.59 \\
(\mathrm{P}<0.0001)\end{array}$ & & & & & & & & \\
\hline $\begin{array}{l}\text { Comprimento da primeira vértebra sacra } \\
\qquad \text { (H) }\end{array}$ & $\begin{array}{c}0.26 \\
(\mathrm{p}=0.0107)\end{array}$ & $\begin{array}{c}0.11 \\
(\mathrm{p}=0.273)\end{array}$ & $\begin{array}{c}0.11 \\
(\mathrm{p}=0.2632)\end{array}$ & $\begin{array}{c}0.02 \\
(\mathrm{p}=0.8245)\end{array}$ & $\begin{array}{c}0.1 \\
(\mathrm{p}=0.3496)\end{array}$ & $\begin{array}{c}0.24 \\
(\mathrm{p}=0.0185)\end{array}$ & $\begin{array}{c}0.18 \\
(\mathrm{p}=0.0814)\end{array}$ & & & & & & & \\
\hline $\begin{array}{l}\text { Largura da primeira vértebra sacra } \\
\text { (I) }\end{array}$ & $\begin{array}{c}0.36 \\
(\mathrm{p}=0.0003)\end{array}$ & $\begin{array}{c}0.07 \\
(\mathrm{p}=0.4627)\end{array}$ & $\begin{array}{c}0.13 \\
(\mathrm{p}=0.1932)\end{array}$ & $\begin{array}{c}0.05 \\
(\mathrm{p}=0.6236)\end{array}$ & $\begin{array}{c}0.17 \\
(\mathrm{p}=0.0956)\end{array}$ & $\begin{array}{c}0.3 \\
(\mathrm{p}=0.0031)\end{array}$ & $\begin{array}{c}0.16 \\
(\mathrm{p}=0.1187)\end{array}$ & $\begin{array}{c}0.75 \\
(\mathrm{P}<0.0001)\end{array}$ & & & & & & \\
\hline $\begin{array}{l}\text { Forame incisivo até o forame palatino maior } \\
\text { - esquerdo } \\
\text { (J) }\end{array}$ & $\begin{array}{c}0.13 \\
(\mathrm{p}=0.214)\end{array}$ & $\begin{array}{c}-0.06 \\
(\mathrm{p}=0.5295)\end{array}$ & $\begin{array}{c}0.12 \\
(\mathrm{p}=0.2449)\end{array}$ & $\begin{array}{c}0.06 \\
(\mathrm{p}=0.5477)\end{array}$ & $\begin{array}{c}0.09 \\
(\mathrm{p}=0.3937)\end{array}$ & $\begin{array}{c}0.12 \\
(\mathrm{p}=0.2584)\end{array}$ & $\begin{array}{c}0.06 \\
(\mathrm{p}=0.5732)\end{array}$ & $\begin{array}{c}0.22 \\
(\mathrm{p}=0.033)\end{array}$ & $\begin{array}{c}0.28 \\
(\mathrm{p}=0.0066)\end{array}$ & & & & & \\
\hline $\begin{array}{l}\text { Forame incisivo até o forame palatino maior } \\
\qquad \begin{array}{l}\text { - direito } \\
\text { (K) }\end{array}\end{array}$ & $\begin{array}{c}0.09 \\
(\mathrm{p}=0.3988)\end{array}$ & $\begin{array}{c}-0.1 \\
(\mathrm{p}=0.3256)\end{array}$ & $\begin{array}{c}0.25 \\
(\mathrm{p}=0.0148)\end{array}$ & $\begin{array}{c}0.08 \\
(\mathrm{p}=0.4376)\end{array}$ & $\begin{array}{c}0.15 \\
(\mathrm{p}=0.1508)\end{array}$ & $\begin{array}{c}0.1 \\
(\mathrm{p}=0.3478)\end{array}$ & $\begin{array}{c}0.05 \\
(\mathrm{p}=0.6397)\end{array}$ & $\begin{array}{c}0.27 \\
(\mathrm{p}=0.007)\end{array}$ & $\begin{array}{c}0.32 \\
(\mathrm{p}=0.0016)\end{array}$ & $\begin{array}{c}0.87 \\
(\mathrm{P}<0.0001)\end{array}$ & & & & \\
\hline $\begin{array}{l}\text { Forame incisivo até o forame palatino maior } \\
\text { - média } \\
\text { (L) }\end{array}$ & $\begin{array}{c}0.11 \\
(\mathrm{p}=0.2841)\end{array}$ & $\begin{array}{c}-0.09 \\
(\mathrm{p}=0.4009)\end{array}$ & $\begin{array}{c}0.19 \\
(\mathrm{p}=0.06)\end{array}$ & $\begin{array}{c}0.07 \\
(\mathrm{p}=0.4747)\end{array}$ & $\begin{array}{c}0.12 \\
(\mathrm{p}=0.2303)\end{array}$ & $\begin{array}{c}0.11 \\
(\mathrm{p}=0.2859)\end{array}$ & $\begin{array}{c}0.06 \\
(\mathrm{p}=0.5952)\end{array}$ & $\begin{array}{c}0.26 \\
(\mathrm{p}=0.0121)\end{array}$ & $\begin{array}{c}0.31 \\
(\mathrm{p}=0.0023)\end{array}$ & $\begin{array}{c}0.96 \\
(\mathrm{P}<0.0001)\end{array}$ & $\begin{array}{c}0.97 \\
(\mathrm{P}<0.0001)\end{array}$ & & & \\
\hline $\begin{array}{l}\text { Básio até o forame palatino maior - esquerdo } \\
\qquad(\mathrm{M})\end{array}$ & $\begin{array}{c}-0.07 \\
(\mathrm{p}=0.5029)\end{array}$ & $\begin{array}{c}0.08 \\
(\mathrm{p}=0.3918)\end{array}$ & $\begin{array}{c}0.23 \\
(\mathrm{p}=0.0159)\end{array}$ & $\begin{array}{c}-0.1 \\
(\mathrm{p}=0.2781)\end{array}$ & $\begin{array}{c}0.01 \\
(\mathrm{p}=0.9092)\end{array}$ & $\begin{array}{c}-0.12 \\
(\mathrm{p}=0.2031)\end{array}$ & $\begin{array}{c}-0.12 \\
(\mathrm{p}=0.2247)\end{array}$ & $\begin{array}{c}-0.01 \\
(\mathrm{p}=0.8859)\end{array}$ & $\begin{array}{c}0.01 \\
(\mathrm{p}=0.9458)\end{array}$ & $\begin{array}{c}-0.22 \\
(\mathrm{p}=0.0276)\end{array}$ & $\begin{array}{c}-0.16 \\
(\mathrm{p}=0.1182)\end{array}$ & $\begin{array}{c}-0.2 \\
(\mathrm{p}=0.0532)\end{array}$ & & \\
\hline $\begin{array}{l}\text { Básio até o forame palatino maior - direito } \\
\qquad(\mathrm{N})\end{array}$ & $\begin{array}{c}0.2 \\
(\mathrm{p}=0.0379)\end{array}$ & $\begin{array}{c}0.25 \\
(\mathrm{p}=0.0101)\end{array}$ & $\begin{array}{c}0.13 \\
(\mathrm{p}=0.1679)\end{array}$ & $\begin{array}{c}0.05 \\
(\mathrm{p}=0.5952)\end{array}$ & $\begin{array}{c}0.2 \\
(\mathrm{p}=0.045)\end{array}$ & $\begin{array}{c}0.27 \\
(\mathrm{p}=0.0042)\end{array}$ & $\begin{array}{c}0.22 \\
(\mathrm{p}=0.0261)\end{array}$ & $\begin{array}{c}-0.06 \\
(\mathrm{p}=0.5324)\end{array}$ & $\begin{array}{c}0.08 \\
(\mathrm{p}=0.4215)\end{array}$ & $\begin{array}{c}0.07 \\
(\mathrm{p}=0.481)\end{array}$ & $\begin{array}{c}0.14 \\
(\mathrm{p}=0.172)\end{array}$ & $\begin{array}{c}0.11 \\
(\mathrm{p}=0.2786)\end{array}$ & $\begin{array}{c}0.17 \\
(\mathrm{p}=0.0735)\end{array}$ & \\
\hline $\begin{array}{l}\text { Básio até o forame palatino maior - média } \\
\qquad(\mathrm{O})\end{array}$ & $\begin{array}{c}0.07 \\
(\mathrm{p}=0.5033)\end{array}$ & $\begin{array}{c}0.2 \\
(\mathrm{p}=0.0378)\end{array}$ & $\begin{array}{c}0.24 \\
(\mathrm{p}=0.0104)\end{array}$ & $\begin{array}{c}-0.05 \\
(\mathrm{p}=0.6196)\end{array}$ & $\begin{array}{c}0.12 \\
(\mathrm{p}=0.2243)\end{array}$ & $\begin{array}{c}0.06 \\
(\mathrm{p}=0.5064)\end{array}$ & $\begin{array}{c}0.04 \\
(\mathrm{p}=0.72)\end{array}$ & $\begin{array}{c}-0.05 \\
(\mathrm{p}=0.6431)\end{array}$ & $\begin{array}{c}0.05 \\
(\mathrm{p}=0.609)\end{array}$ & $\begin{array}{c}-0.12 \\
(\mathrm{p}=0.2412)\end{array}$ & $\begin{array}{c}-0.04 \\
(\mathrm{p}=0.7279)\end{array}$ & $\begin{array}{c}-0.08 \\
(\mathrm{p}=0.4427)\end{array}$ & $\begin{array}{c}0.83 \\
(\mathrm{P}<0.0001)\end{array}$ & $\begin{array}{c}0.69 \\
(\mathrm{P}<0.0001)\end{array}$ \\
\hline
\end{tabular}


Analisando as Tabelas 3 e 4, observa-se que a correlação entre o diâmetro vertical do acetábulo com o comprimento do forame obturado foi afetada pelo sexo, pois a correlação foi importante apenas para o sexo feminino. Foi ainda reduzida para o sexo feminino a correlação entre o diâmetro vertical do acetábulo com a profundidade da incisura isquiática maior, mas o sexo não afetou a relação entre a largura superior sacro com o diâmetro vertical do acetábulo ou com a profundidade da incisura isquiática maior.

Tabela 5. Modelo de regressão logística para determinação do sexo, utilizando como variáveis as médias de medidas realizadas.

\begin{tabular}{lccccc}
\hline & Beta & Erro padrão & Wald & Valor de p & Exp(Beta) \\
\hline Diâmetro vertical do acetábulo & 0.45 & 0.09 & 25.4 & $<0.0001$ & 1.6 \\
Comprimento do forame obturado & 0.19 & 0.06 & 9.5 & 0.0021 & 1.26 \\
Incisura isquiática maior - Corda & -0.04 & 0.03 & 2.3 & 0.12 & 0.96 \\
Básio até o forame palatino maior - média & -0.14 & 0.06 & 5.8 & 0.0156 & 0.87 \\
Constante & -21.1 & 5.05 & 17.5 & $<0.0001$ & $6.7 \times 10-10$ \\
\hline
\end{tabular}

Considerando a probabilidade de acerto ao acaso, os dados revelaram uma porcentagem de 57,5\% de chance de acertar o sexo. A regressão revelou que o melhor modelo (Tabela 5) foi aquele composto pelas medidas do diâmetro vertical do acetábulo, comprimento do forame obturado, corda da incisura isquiática maior e média entre os lados esquerdo e direito do básio até o forame palatino maior (Qui-quadrado=96.1, $\mathrm{p}<0.0001$ ). As outras medidas não foram importantes para o modelo $(\mathrm{p}>0.05)$. $O \mathrm{R}^{2}$ de Nagelkerke mostrou que as variáveis são responsáveis por $59,6 \%$ da variação encontrada no sexo.

Assim, a regressão logística seria:

Sexo $=-21.1+(0.45 \times$ Diâmetro vertical do acetábulo $)+(0.19 \times$ Comprimento do forame obturado $)-$ $(0.04 \times$ Corda da incisura isquiática maior $)-(0.14 \times$ média do básio até o forame palatino maior $)$.

Tabela 6. Relação entre a predição do sexo pela fórmula de regressão logística e o sexo real.

\begin{tabular}{|c|c|c|c|c|}
\hline & & \multicolumn{3}{|c|}{ Predição pela fórmula } \\
\hline & & Feminino & Masculino & Percentagem correta \\
\hline \multirow[t]{3}{*}{ Sexo real } & Feminino & 55 & 13 & 80.9 \\
\hline & Masculino & 10 & 82 & 89.1 \\
\hline & & & Porcentagem geral correta & 85.7 \\
\hline
\end{tabular}

A Tabela 6 revela que, aplicada a fórmula de regressão logística na amostra, houve a correta identificação de sexo no percentual de $85,7 \%$, a revelar a sua acurácia para a predição do sexo. 


\section{Discussão}

O presente estudo proporciona dados para permitir a identificação do sexo em esqueletos brasileiros, de predominância étnica faioderma e leocoderma.

Deve-se deixar claro que, de acordo com Penna (2002), ${ }^{7}$ no Brasil, o indivíduo leucoderma e o indivíduo faioderma possuem $30 \%$ de contribuição africana, 30\% europeia e 30\% ameríndia, e a principal situação fenotípica que os distingue é a cor da pele.

$\mathrm{Na}$ presente pesquisa, foram analisadas 191 ossadas para se observar a viabilidade das medidas obtidas do osso pélvico, da base do crânio e da primeira vértebra sacral na identificação do sexo, sendo que os resultados obtidos permitem concluir pela existência de dimorfismo sexual nas medidas ósseas.

Nas medidas realizadas no crânio, três apresentaram dimorfismo sexual: a do forame incisivo até o forame palatino maior esquerdo, forame incisivo até o forame palatino maior direito e básio até o forame palatino maior esquerdo, corroborando com os achados de Mahakkanukrauh. ${ }^{8}$

Por sua vez, as medidas que apresentaram dimorfismo sexual relevante realizadas na pelve e no sacro foram: diâmetro vertical do acetábulo, comprimento do forame obturado e profundidade da incisura isquiática maior e largura superior do sacro.

Assim, nesta parte, o estudo demonstra que o dimorfismo sexual pode ser identificado não somente em medidas feitas no crânio, mas também em outros ossos, tal qual consta na literatura, em estudos realizados na mandíbula, ${ }^{9}$ patela, ${ }^{10}$ osso esterno ${ }^{11}$ e também em ossos longos, como a tíbia ${ }^{12}$ e o úmero. ${ }^{13}$
Para fins de identificação do sexo, a relevância é maior ainda quando as medidas são utilizadas de forma combinada. Com as variáveis diâmetro vertical do acetábulo, comprimento do forame obturado, corda da incisura isquiática maior e básio até o forame palatino maior, obteve-se fórmula em regressão logística que permite índice $85,7 \%$ de identificação do sexo. Comparado com a média entre $70 \%$ e $95 \%$ obtida por autores em medidas craniométricas, ${ }^{14,15}$ pode-se inferir que o percentual obtido é muito bom, de forma a se afirmar da viabilidade da utilização dos ossos crânio, sacro e pelve como método quantitativo para a identificação do sexo post mortem em ossadas brasileiras, consistindo na prática, em mais uma ferramenta na área pericial.

\section{CONSIDERAÇÕES FINAIS}

Dos resultados obtidos no presente trabalho, pode-se concluir que:

1 - As medidas feitas no crânio (forame incisivo até o forame palatino maior esquerdo, forame incisivo até o forame palatino maior direito e básio até o forame palatino maior esquerdo), sacro (largura superior) e pelve (diâmetro vertical do acetábulo, comprimento do forame obturado e profundidade da incisura isquiática maior) apresentam dimorfismo sexual;

2 - Utilizando-se as medidas: diâmetro vertical do acetábulo, comprimento do forame obturado, corda da incisura isquiática maior e básio até o forame palatino maior obteve-se fórmula em regressão logística que atinge índice de identificação do sexo de 85,7\%.

\section{REFERÊNCIAS}

1. Saccucci M., Cipriani F., Carderi S., Di Carlo G., D’Attilio M., Rodolfino D., Festa F., Polimeni A. Gender assessment through three-dimensional analysis of maxillary sinuses by means of cone beam computed tomography. Eur Rev Med Pharmacol Sci. 2015; 19 (2): 185-93.

2. Weston EM, Friday AE, Liò P. Biometric evidence that sexual selection has shaped the hominin face. PLoS One. 2007 Aug. 8 ;2 (8): e710.

3. Zaki ME, Soliman MA, El-Bassyouni HT. A cephalometric study of skulls from the Bahriyah oasis. J Forensic Dent Sci. 2012 Jul; 4 (2): 88-92. doi: 10.4103/0975-1475.109895.

4. Kesterke MJ, Raffensperger ZD, Heike CL, Cunningham ML, Hecht JT, Kau CH, Nidey NL, Moreno LM, Wehby GL, Marazita ML, Weinberg SM. Using the 3D Facial Norms Database to investigate craniofacial sexual dimorphism in healthy children, adolescents, and adults. Biol Sex Differ. 2016 Apr 22; 7: 23.

5. Coma JMR. Antropologia Forense, Madrid: Ministério de Justicia - Centro de Publicaciones, 1999, 178-185; 569-583 e 604-607. 
6. Iscan YM. Forensic anthropology of sex and body size, Forens Sci Int. 2005147, p. 107-112.

7. Penna, SDJ. (Org.) (2002). Homo brasilis: Genetic, Linguistic, Historical and Socioanthropological Aspects of the Brazilian People's Formation. Ribeirão Preto, FUNPEC.

8. Mahakkanukrauh P., Sinthubua A., Prasitwattanaseree S., Ruengdit S., Singsuwan P., Praneatpolgrang S., Duangto P. Craniometric study for sex determination in a Thai population. Anat Cell Biol. 2015 Dec; 48 (4): 275-83.

9. Wankhede KP, Bardale RV, Chaudhari GR, Kamdi NY. Determination of sex by discriminant function analysis of mandibles from a Central Indian population. J Forensic Dent Sci. 2015 Jan-Apr, 7 (1): 37-43.

10. Dayal MR, Bidmos MA. Discriminating sex in South African blacks using patella imensions. J Forensic Sci. 2005 Nov, 50 (6): $1.294-7$.

11. Ekizoglu O., Hocaoglu E., Inci E., Bilgili MG, Solmaz D., Erdil I., Can IO. Sex estimation from sternal measurements using multidetector computed tomography. Medicine (Baltimore), 2014 Dec, 93 (27): e240.

12. Slaus M., Bedić Z., Strinović D., Petrovečki V. Sex determination by discriminant function analysis of the tibia for contemporary Croats. Forensic Sci Int. 2013 Mar 10; 226 (1-3): 302 e1-4.

13. Bašić Ž., Anterić I., Vilović K., Petaros A., Bosnar A., Madžar T., Polašek O., Anđelinović Š. Sex determination in skeletal remains form the medieval Eastern Adriatic coast - discriminant function analysis of humeri. Croat Med J. 2013 Jun, 54 (3): 272-8.

14. Amin, W., Otham, D. Gender Dimorphism of Crania in Young Jordanian Adults: Discriminant Function Analysis Approach for Gender Prediction. J Current Sur. 2014 Sep, 4 (3), 76-85.

15. Asghar A., Dixit A., Rani M. Morphometric Study of Nasal Bone and Piriform Aperture in Human Dry Skull of Indian Origin. J Clin Diagn Res. 2016, Jan; 10 (1): AC05-7.

\section{DADOS DOS AUTORES}

\section{Graciele Dib Nunes Silva}

Graduada em Odontologia pela Faculdade de Odontologia de Piracicaba (UNICAMP). Piracicaba/SP - Brasil. joaobedran@ig.com.br

\section{Cristiane Martins Schmidt}

Graduada em Odontologia pela Faculdade de Odontologia de Piracicaba (UNICAMP). Piracicaba/SP - Brasil. joaobedran@ig.com.br

\section{VIVIANe UlBRICHT}

Doutoranda em Anatomia na Faculdade de Odontologia de Piracicaba (UNICAMP). Coordenadora Local dos Cursos de Extensão na mesma instituição de Perícias Judiciais e "AD HOC" e Odontologia do Esporte. Perita Judicial em várias comarcas de São Paulo e interior de São Paulo. Piracicaba/SP - Brasil. joaobedran@ig.com.br

\section{Francisco Carlos Groppo}

Doutor em Odontologia pela Faculdade de Odontologia de Piracicaba (UNICAMP). Professor Titular na mesma instituição. Piracicaba/SP - Brasil. joaobedran@ig.com.br

\section{EDUARDo DARUge JUNior}

Doutor em Radiologia Odontologica pela Faculdade de Odontologia de Piracicaba (UNICAMP). Professor Livre Docente na mesma instituição. Piracicaba/SP - Brasil. joaobedran@ig.com.br

\section{Luiz Francesquini Junior}

Doutor em Clínica Odontológica e Doutor em Radiologia Odontológica pela Universidade Estadual de Campinas. Professor Livre Docente Associado na mesma instituição. Piracicaba/SP - Brasil. francesq@unicamp.br

\section{João Cesar barbieri Bedran de Castro}

Mestre em Direito pela Pontifícia Universidade Católica de São Paulo (PUC) e em Biologia Buco-Dental pela Faculdade de Odontologia de Piracicaba (UNICAMP). Procurador do Estado. Piracicaba/SP - Brasil. joaobedran@ig.com.br

Submetido em: 24-4-2018

Aceito em: 3-10-2019 